\title{
Structural equation modeling of approval voting data
}

\author{
Rung-ChING TsaI \\ National Taiwan Normal University, Taipei, Taiwan
}

\begin{abstract}
The method of approval voting is a commonly used voting procedure in which each judge selects a subset of the alternatives. By postulating that the random utilities associated with the choice options in approval voting elections follow a multivariate normal distribution under the Thurstonian framework, Regenwetter, Ho, and Tsetlin (2007) attempted to integrate the normative theories and individual variabilities in modeling social behavior. However, their approach is limited to only three alternatives, due to computational intractability as the number of alternatives increases. In this article, we reparameterize extensions of their models under the structural equation modeling framework and propose the use of limited information methods for estimating model parameters. As a result, we are able to extend their previous approach to the analysis of approval voting data with any number of alternatives. Two applications are presented to illustrate the usefulness of such an approach.
\end{abstract}

The method of approval voting is a commonly used procedure even in situations that are not normally considered as competitions. For example, although early stages of a job recruitment process are about qualifications for the position, it is common practice for a recruitment committee to apply approval voting to determine the qualification of the candidates. In this approach, each judge selects a subset of candidates that are deemed appropriate. Most studies on approval voting have focused either on constructing normative theories or on finding heuristics to describe the decision process. Few studies have investigated real empirical voting behavior for the adequacy or suitability of those proposals (Falmagne \& Regenwetter, 1996; Marley, 1993, 2002; Regenwetter \& Grofman, 1998; Takane, 1998). Falmagne and Regenwetter proposed an empirically testable probabilistic model for approval voting data without making assumptions about particular parametric families for the distribution of the random utilities associated with the alternatives in the choice set. Regenwetter and Grofman further applied the Falmagne and Regenwetter random utility model to the analysis of 10 election data sets and concluded that approval voting gives rise to outcomes that are consistent with sincere voting outcomes under various normative social choice benchmarks.

In understanding and forecasting voting behavior in electoral research, multistage process models have also been shown to provide good forecasts (Paap et al., 2005; Tillie, 1995; van der Eijk, van der Burg, Kroh, \& Franklin, 2006). The multistage process usually includes a consideration set formation stage at which a presumably smaller subset of choice options is elicited from the set of all options and used for the final voting decision process. The inclusion of such a consideration set stage seems to recon- struct the actual voting process, because voters are likely to discard inconceivable alternatives so that they have fewer choice options as time approaches the election. In addition to political science, consideration set models are becoming increasingly popular for modeling consumer behavior in marketing research (Bian \& Moutinho, 2009; Eliaz \& Spiegler, 2009; Haubl \& Trifts, 2000; Punj \& Moore, 2009; Shapiro, Macinnis, \& Heckler, 1997; Shocker, BenAkiva, Boccara, \& Nedungadi, 1991).

The consideration set formulation is almost identical to the approval voting task because in both cases, the voter is thought to choose or approve a subset of options from the set of all alternatives. Their main difference simply falls on the fact that the consideration set might not be explicitly observed in the multistage model, whereas approval voting data are usually directly observed. Because of their similarity in task, approval voting models can be considered as possible alternatives for the consideration set formulation. On the other hand, consideration set models might be useful for modeling approval voting behavior. For example, Paap et al. (2005) have presented a two-stage model where the inclusion of a candidate in the consideration set is described using a simple multivariate probit model with covariates of the voters as the explanatory variables. Given the consideration set, a party is chosen from the consideration set if its respective utility exceeds that of others by a threshold in the voting decision stage.

Similar to the use of a probit model for consideration set formulation, Regenwetter et al. (2007) proposed the distributional assumption of normality for the random utilities associated with the alternatives in approval voting and attempted to integrate normative theories and individual variabilities to model social behavior under a Thurstonian

R.-C.Tsai, rtsai@math.ntnu.edu.tw 
framework. However, unlike consideration set models in which all the options are allowed to enter the consideration set, the approval voting models proposed by Regenwetter et al. permit neither the all nor the none response patterns and, therefore, provide only part of the story in individual difference in the judges' decision heuristics. Here, we further extend the Regenwetter et al. model to allow for the possibility of both the all and none voting patterns, and consequently, the resultant approval voting model could be used for the consideration set formulation as well.

In estimating model parameters for approval voting models, the full information maximum-likelihood estimation method via numerical integrations would become infeasible and cumbersome as the number of alternatives increases. As a result, most applications of probabilistic models for approval voting were limited to choice sets of three or four alternatives. In an analysis of paired comparison and ranking data, Tsai and $\mathrm{Wu}$ (2004) and Maydeu-Olivares and Böckenholt (2005) showed that the Thurstonian preference models can be formulated as structural equation models (SEMs), and consequently, software packages such as Mx and Mplus can be used for estimation of model parameters. We will show that it is also straightforward to reformulate an existing model for approval voting using an SEM framework and, by doing so for complete data, we hope to facilitate not only future applications, but also more extensions with the advance of SEM techniques.

In this article, our goal is to introduce a formulation for the approval voting mechanism undertaken in the choice process under the SEM framework and, therefore, provide a means to better understand voting behavior. Consequently, we are able to extend previous approaches to the analysis of approval voting data with any number of alternatives. The remainder of this article is structured as follows. First, we briefly review the model of approval voting data proposed by Regenwetter et al. (2007) and its extensions. Second, we formulate these models under an SEM framework. In addition to estimation methods associated with the present approach, two applications are presented to illustrate its usefulness for understanding choice behavior.

\section{APPROVAL VOTING MODELS}

Regenwetter et al. (2007) translated the prescriptive rule for approval voting of Merrill (1979) and Brams and Fishburn (1983) into a probabilistic choice model. In the probabilistic random utility model for the underlying mechanism of the approval voting process, it is assumed that each voter first generates his or her utilities of the candidates, and then, his or her subjective assessments that any given candidate(s) will get elected are determined by whether the utilities associated with the particular candidates exceed the average utility of all the candidates in the choice set. In other words, the probability that a randomly chosen voter approves of the subset $A$, denoted as $P_{A}$, under Regenwetter et al. is computed as the ignorance prior random utility model (IP-RUM) of sophisticated approval voting

$$
P_{A}=P\left(\min _{r \in A} U_{i r} \geq \frac{\sum_{j=1}^{J} U_{i j}}{J}>\max _{t \notin A} U_{i t}\right),
$$

where $A$ is a subset of the set of all candidates in the choice set $C$, with the number of candidates $|C|=J$, and $U_{i j}$ denotes the random variable of voter $i$ 's random utility for candidate $C_{j}$.

This approval voting mechanism implies that the average utility

$$
\frac{\sum_{j=1}^{J} U_{i j}}{J}
$$

is used as an "internal" threshold in determining whether any particular candidate is approved. Here, we introduce a reparameterization of the model above to ease a computational difficulty encountered in Regenwetter et al. (2007). The deviation to the average utility, $X_{i j}$, is defined as follows:

$$
X_{i j}=U_{i j}-\frac{\sum_{j=1}^{J} U_{i j}}{J},
$$

and

$$
C_{j} \text { is chosen by judge } i \text { if and only if } X_{i j} \geq 0,
$$

where we have $\Sigma_{j=1}^{J} X_{i j}=0$.

The approval voting data can be summarized by the response vector $\mathbf{W}$, which contains the binary approval voting outcomes $\left(\mathbf{W}_{1}, \mathbf{W}_{2}, \ldots, \mathbf{W}_{n}\right)$. When judge $i$ chooses candidate $C_{j}$ in the choice set, $W_{i j}=1$, and 0 otherwise. The choice is determined by the sign of the latent judgment $Y_{i j}$ of candidate $C_{j}$ for judge $i$ such that

$$
W_{i j}=\left\{\begin{array}{l}
1, Y_{i j} \geq 0, \\
0, Y_{i j}<0,
\end{array}\right.
$$

where

$$
Y_{i j}=\left\{\begin{array}{cl}
X_{i j} & , j=1,2, \ldots, J-1, \\
X_{i J}=-X_{i 1}-X_{i 2} & \\
-\cdots-X_{i, J-1} & , j=J .
\end{array}\right.
$$

The latent judgments can be written conveniently as a linear model. For example, in a voting task with candidates $C_{1}, C_{2}, C_{3}$, and $C_{4}$ (i.e., $J=4$ ), we obtain

$$
\begin{aligned}
\mathbf{Y}_{i}=\left(\begin{array}{l}
Y_{i 1} \\
Y_{i 2} \\
Y_{i 3} \\
Y_{i 4}
\end{array}\right) & =\left(\begin{array}{cccc}
1-\frac{1}{4} & -\frac{1}{4} & -\frac{1}{4} & -\frac{1}{4} \\
-\frac{1}{4} & 1-\frac{1}{4} & -\frac{1}{4} & -\frac{1}{4} \\
-\frac{1}{4} & -\frac{1}{4} & 1-\frac{1}{4} & -\frac{1}{4} \\
-\frac{1}{4} & -\frac{1}{4} & -\frac{1}{4} & 1-\frac{1}{4}
\end{array}\right)\left(\begin{array}{l}
U_{i 1} \\
U_{i 2} \\
U_{i 3} \\
U_{i 4}
\end{array}\right) \\
& =\left(\begin{array}{ccc}
1 & 0 & 0 \\
0 & 1 & 0 \\
0 & 0 & 1 \\
-1 & -1 & -1
\end{array}\right)\left(\begin{array}{l}
X_{i 1} \\
X_{i 2} \\
X_{i 3}
\end{array}\right)=\mathbf{A} \mathbf{X}_{i(-4)},
\end{aligned}
$$

where $\mathbf{X}_{i(-4)}=\mathbf{X}_{i(-J)}$ is the $(J-1)$-dimensional vector containing the deviation between the utility of each of the 
first $J-1$ candidates and the average utility of all $J$ candidates by person $i$, and $\mathbf{A}$ is a design matrix describing the comparison structure, such that

$$
\mathbf{A}_{J \times(J-1)}=\left[\begin{array}{l}
\mathbf{I}_{J-1} \\
(-1) \mathbf{1}^{\prime}
\end{array}\right] .
$$

It can be easily seen that, while assuming the latent continuum of the utilities under the approval voting formulation in Equation 1, neither the all nor the none response pattern would occur with positive probability. In other words, such models do not account for choices of full or empty sets, and consequently, data on such ballots are usually left out of the data analysis, despite the fact that these two patterns are consistently observed across many approval voting tasks. The additional occasion-specific random component $\epsilon_{i j}$ is introduced into the judgment process to allow for the possibility of both the all and the none choice patterns such that, instead of Equation 2, we have for the latent response

$$
Y_{i j}=U_{i j}-\frac{\sum_{j=1}^{J} U_{i j}}{J}+\epsilon_{i j},
$$

where $\epsilon$ is meant to represent randomness unaccounted for by the greater than average mechanism. We can imagine that, even with the same candidate, it is possible that the approval voting responses might vary from occasion to occasion. In other words, the occasion-specific random component would be most meaningful and systematically investigated through repeated evaluations of the same candidates. As a result, we have for the case of $J=4$,

$$
\begin{aligned}
\mathbf{Y}_{i} & =\left(\begin{array}{c}
Y_{i 1} \\
Y_{i 2} \\
Y_{i 3} \\
Y_{i 4}
\end{array}\right) \\
& =\left(\begin{array}{cccc}
1-\frac{1}{4} & -\frac{1}{4} & -\frac{1}{4} & -\frac{1}{4} \\
-\frac{1}{4} & 1-\frac{1}{4} & -\frac{1}{4} & -\frac{1}{4} \\
-\frac{1}{4} & -\frac{1}{4} & 1-\frac{1}{4} & -\frac{1}{4} \\
-\frac{1}{4} & -\frac{1}{4} & -\frac{1}{4} & 1-\frac{1}{4}
\end{array}\right)\left(\begin{array}{l}
U_{i 1} \\
U_{i 2} \\
U_{i 3} \\
U_{i 4}
\end{array}\right)+\left(\begin{array}{c}
\epsilon_{i 1} \\
\epsilon_{i 2} \\
\epsilon_{i 3} \\
\epsilon_{i 4}
\end{array}\right) \\
& =\left(\begin{array}{ccc}
1 & 0 & 0 \\
0 & 1 & 0 \\
0 & 0 & 1 \\
-1 & -1 & -1
\end{array}\right)\left(\begin{array}{l}
X_{i 1} \\
X_{i 2} \\
X_{i 3}
\end{array}\right)+\left(\begin{array}{l}
\epsilon_{i 1} \\
\epsilon_{i 2} \\
\epsilon_{i 3} \\
\epsilon_{i 4}
\end{array}\right)=\mathbf{A} \mathbf{X}_{i(-4)}+\boldsymbol{\epsilon},
\end{aligned}
$$

where $\mathbf{X}_{i(-4)}$ and $\mathbf{A}$ are similarly defined as above. Although the modification of adding the occasion-specific randomness term $\epsilon$ seems minor, the model can now allow for the none and all responses under such an underlying mechanism. We can further assume that occasion-specific error terms $\epsilon \mathrm{s}$ are independent with zero means and a diagonal covariance matrix $\boldsymbol{\Sigma} \epsilon=\Psi$. Under this model, the probability of observing the none pattern $\left(W_{1}, W_{2}, W_{3}, W_{4}\right)=$ $(0,0,0,0)$ for judge $i$ with $J=4$ can be derived as the equation at the bottom of the page. The probability of observing the all response $\left(W_{1}, W_{2}, W_{3}, W_{4}\right)=(1,1,1,1)$ can be similarly derived. That is, the none and all responses are admissible for an individual voter.

To characterize the individual difference in voters' perceived utilities of the alternatives, $U_{1}, U_{2}, \ldots, U_{J}$ are assumed to be normally distributed with mean vector $\boldsymbol{\mu}_{u}$ and $\boldsymbol{\Sigma}_{u}$ - that is, $\mathbf{U} \sim \mathcal{N}\left(\boldsymbol{\mu}_{u}, \boldsymbol{\Sigma}_{u}\right)$. In addition, the occasionspecific randomness terms $\epsilon_{1}, \epsilon_{2}, \ldots, \epsilon_{J}$ are also assumed to be normal such that $\epsilon \sim \mathcal{N}(\mathbf{0}, \mathbf{I})$. With an additional independence assumption between $\mathbf{U}$ and $\boldsymbol{\epsilon}$, the resultant latent judgment $\mathbf{Y}$ will follow a multivariate normal distribution with mean vector

$$
\boldsymbol{\mu}_{Y}=\left(\mathbf{I}-\frac{1}{J} \mathbf{1 1}^{\prime}\right) \boldsymbol{\mu}_{u}=\mathbf{A} \boldsymbol{\mu}_{X(-J)}
$$

and covariance matrix

$$
\boldsymbol{\Sigma}_{Y}=\left(\mathbf{I}-\frac{1}{I} \mathbf{1 1 ^ { \prime }}\right) \boldsymbol{\Sigma}_{u}\left(\mathbf{I}-\frac{1}{I} \mathbf{1 1 ^ { \prime }}\right)+\boldsymbol{\Sigma}_{\epsilon}=\mathbf{A} \boldsymbol{\Sigma}_{X(-J)} \mathbf{A}^{\prime}+\mathbf{I} .
$$

The simple covariance structure imposed on $\epsilon$ that $\boldsymbol{\Sigma}_{\epsilon}=\mathbf{I}$ is partly due to the lack of repeated measures in an approval voting task in obtaining more reliable estimates of $\boldsymbol{\Sigma}_{\epsilon}$. This homogeneity assumption induces an equality in the probabilities of the all and none patterns and, therefore, often provides a poor fit to complete data with asymmetric probabilities in these two responses (Tsai \& Böckenholt, 2006). To better characterize the underlying judgment process giving rise to the approval voting data, an "external" threshold, $\delta$, could be introduced to account for an asymmetry in the all and none response probabilities such that, instead of using zero as the threshold in Equation 4, we have

$$
W_{i j}= \begin{cases}1, & Y_{i j} \geq \delta, \\ 0, & Y_{i j}<\delta,\end{cases}
$$

where $\delta$ is a nonzero threshold for the latent judgment $Y_{i j}$ upon determining whether $C_{j}$ is chosen by judge $i$. That is, the latent judgment $Y_{i j}=X_{i j}+\boldsymbol{\epsilon}_{i j}$ needs to exceed the external threshold $\delta$ to give rise to the response $W_{i j}=1$.

$$
\begin{aligned}
P\left[\left(W_{1}, W_{2}, W_{3}, W_{4}\right)\right. & \left.=(0,0,0,0) \mid u_{i 1}, u_{i 2}, u_{i 3}, u_{i 4}\right]=P\left[\mathbf{W}=(0,0,0,0) \mid x_{i 1}, x_{i 2}, x_{i 3}\right] \\
& =P\left(x_{i 1}+\epsilon_{i 1}<0, x_{i 2}+\epsilon_{i 2}<0, x_{i 3}+\epsilon_{i 3}<0,-x_{i 1}-x_{i 2}-x_{i 3}+\epsilon_{i 4}<0 \mid x_{i 1}, x_{i 2}, x_{i 3}\right. \\
& =P\left(x_{i 1}+\epsilon_{i 1}<0\right) P\left(x_{i 2}+\epsilon_{i 2}<0\right) P\left(x_{i 3}+\epsilon_{i 3}<0\right) P\left(-x_{i 1}-x_{i 2}-x_{i 3}+\epsilon_{i 4}<0\right) \\
& =\int_{-\infty}^{-x_{i 1}} f\left(\epsilon_{i 1}\right) d \epsilon_{i 1} \int_{-\infty}^{-x_{i 2}} f\left(\epsilon_{i 2}\right) d \epsilon_{i 2} \int_{-\infty}^{-x_{i 3}} f\left(\epsilon_{i 3}\right) d \epsilon_{i 3} \int_{-\infty}^{x_{i 1}+x_{i 2}+x_{i 3}} f\left(\epsilon_{i 4}\right) d \epsilon_{i 4} \\
& =\Phi\left(\frac{-x_{i 1}}{\sqrt{\psi_{1}}}\right) \Phi\left(\frac{-x_{i 2}}{\sqrt{\psi_{2}}}\right) \Phi\left(\frac{-x_{i 3}}{\sqrt{\psi_{3}}}\right) \Phi\left(\frac{x_{i 1}+x_{i 2}+x_{i 3}}{\sqrt{\psi_{4}}}\right) .
\end{aligned}
$$


In summary, the two types of models considered here are summarized as follows:

1. $\mathbf{Y}_{i}=\mathbf{A} \mathbf{X}_{i(-J)}+\boldsymbol{\epsilon}_{i}$ with a threshold value $\delta=0$,

2. $\mathbf{Y}_{i}=\mathbf{A} \mathbf{X}_{i(-J)}+\epsilon_{i}$ with a nonzero threshold value $\delta$.

Although the conceptual formulation of Equation 1 in Regenwetter et al. (2007) is rather straightforward and so are its extensions, past studies limited their model testing to ballot data with three candidates, due to increasing computational demands as the number of candidates increases. We will show that by formulating their models under the SEM framework, we can extend the applications of these models to approval voting data with an arbitrary number of candidates.

\section{SEM FORMULATIONS}

In this section, we describe how to embed the abovementioned approval voting models under a Thurstonian framework within an SEM framework. To model the linear relations of $J$ indicators $\mathbf{Y}$ on the latent variables $\mathbf{U}$ or $\mathbf{X}_{(-J)}$ and $\epsilon$, the following measurement and structure models with mean structure are commonly used (Jöreskog \& Sörbom, 1996; L. K. Muthén \& Muthén, 1998/2007).

\section{Measurement Model With Mean Structure}

In SEM, each latent construct or variable is usually associated with multiple measures or indicators through a factor analytic measurement model. That is, each latent construct is modeled as a common factor underlying the associated measures

$$
\mathbf{Y}=\boldsymbol{v}+\Lambda_{y} \boldsymbol{\eta}+\boldsymbol{\epsilon}
$$

where $\boldsymbol{v}$ is the $J$-dimensional vector of intercepts or thresholds, $\Lambda_{y}$ is the matrix of factor loadings, and $\epsilon$ is the measurement error associated with $\mathbf{Y}$. The covariance matrix of $\epsilon$ is commonly denoted as $\Theta_{\epsilon}$. For simplicity and identification purpose, we assume $\Theta_{\epsilon}=\mathbf{I}$. In our approval voting model, the factor loadings that describe the association between the latent utilities $\boldsymbol{\eta}=\mathbf{X}_{(-J)}$ and the latent judgment $\mathbf{Y}$ is prespecified in matrix $\mathbf{A}$ such that $\Lambda_{y}=\mathbf{A}$ and for the threshold parameter $\delta, \boldsymbol{v}=-\delta \mathbf{1}$ with either $\delta=0$ or $\delta \neq 0$.

\section{Structural Model With Mean Structure}

In SEM, the structural model describes the relationships among the latent constructs. In the structural models with mean structure, latent constructs or variables are allowed to have nonzero means. In other words,

$$
\eta=\kappa+\mathbf{B}(\boldsymbol{\eta}-\boldsymbol{\kappa})+\boldsymbol{\zeta}
$$

where $\boldsymbol{\kappa}$ is the mean vector of $\boldsymbol{\eta}, \mathbf{B}$ is the matrix of slopes for regressions of latent variables on other latent variables, $\boldsymbol{\zeta}$ is the vector of residuals or deviations, and its covariance matrix is usually denoted as $\boldsymbol{\Psi}$. In our approval voting model, we have the simplest case that $\mathbf{B}=\mathbf{0}$ and $\boldsymbol{\kappa}=$ $\boldsymbol{\mu}_{X(-J)}$.
It is assumed that $\boldsymbol{\epsilon}$ and $\boldsymbol{\zeta}$ both have mean $\mathbf{0}$ and are independent. With the above formulation, the mean and variance matrices of $\mathbf{Y}$ are

$$
\boldsymbol{\mu}_{Y}=\boldsymbol{v}+\Lambda_{y}(\mathbf{I}-\mathbf{B})^{-1} \boldsymbol{\kappa}=\mathbf{A} \boldsymbol{\mu}_{X(-J)}-\delta \mathbf{1},
$$

and

$$
\begin{aligned}
\boldsymbol{\Sigma}_{y} & =\boldsymbol{\Lambda}_{y}(\mathbf{I}-\mathbf{B})^{-1} \mathbf{\Psi}(\mathbf{I}-\mathbf{B})^{-1} \boldsymbol{\Lambda}_{y}^{\prime}+\mathbf{\Theta}_{\boldsymbol{\epsilon}} \\
& =\mathbf{A} \boldsymbol{\Sigma}_{X(-J)} \mathbf{A}^{\prime}+\mathbf{I} .
\end{aligned}
$$

Figure 1 graphically depicts the SEM representation of the approval voting model above for the case of four candidates in the choice set. The only difference between the models with or without an account for the all and none responses would be that $\boldsymbol{\Theta}_{\epsilon}=\operatorname{diag}\left(\theta_{\epsilon 1}, \theta_{\epsilon 2}, \theta_{\epsilon 3}, \theta_{\epsilon 4}\right)=\mathbf{0}$ is fixed to zero for the latter in Figure 1. Note that we do not directly observe the four $Y_{i j}$ variables. Instead, in our data, we have the dichotomization of $Y_{i j}$ - that is, $W_{i j}$-which is determined by the sign of $Y_{i j}$, indicating whether candidate $C_{j}$ is approved by judge $i$.

\section{Threshold and Tetrachoric Correlations Implied by the Models}

Because of the discrete nature of the binary responses in approval voting data, the identified parameters of these models are simply the thresholds and tetrachoric correlations of the underlying latent responses, not necessarily the mean and covariance structures of the latent utilities. To obtain the thresholds and tetrachoric correlations for the approval voting models, it is necessary to standardize the latent judgment $\mathbf{Y}$. Let $\mathbf{D}=\left[\operatorname{Diag}\left(\boldsymbol{\Sigma}_{y}\right)\right]^{-1 / 2}$ denote the diagonal matrix with the reciprocals of the standard deviations of $\mathbf{Y}$. We have for the standardized latent responses $\mathbf{Z}_{y}=\mathbf{D}\left[\mathbf{Y}-\left(\mathbf{A} \boldsymbol{\mu}_{X(-J)}-\delta \mathbf{1}\right)\right]$, its mean $\boldsymbol{\mu}_{\mathbf{Z}}=\mathbf{0}$, and correlation matrix $\mathbf{P}_{\mathbf{Z}_{y}}=\mathbf{D} \boldsymbol{\Sigma}_{y} \mathbf{D}=\mathbf{D}\left(\mathbf{A} \boldsymbol{\Sigma}_{X(-J)} \mathbf{A}+\mathbf{I}\right) \mathbf{D}$.

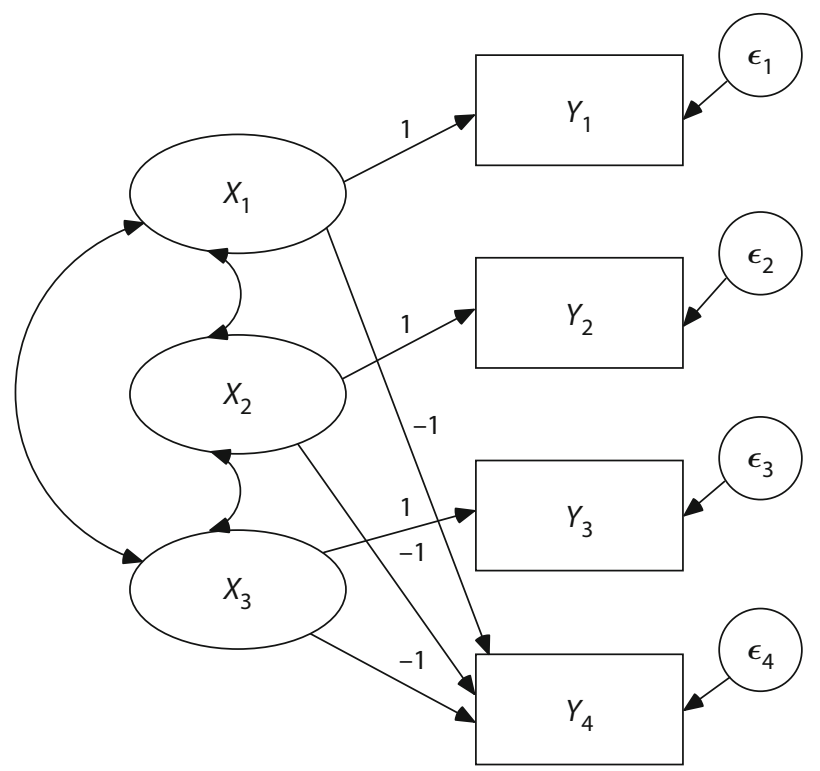

Figure 1. Covariance structure of the random utility models for approval voting data with four alternatives. 
The standardized latent responses $\mathbf{Z}_{y}$ are related to the observed binary approval responses $\mathbf{W}$ via the threshold relationship for each judgment for candidate $C_{j}$,

$$
W_{i j}= \begin{cases}1, & Z_{y_{i j}} \geq \tau_{j}, \\ 0, & Z_{y_{i j}}<\tau_{j},\end{cases}
$$

where $\tau=-\mathrm{D}\left(\mathrm{A} \mu_{\mathrm{X}(-J)}-\delta 1\right)$.

\section{Identification Constraints}

It is well known that because of the comparative nature of the data, not all the parameters in $\boldsymbol{\mu}_{\mu}$ and $\boldsymbol{\Sigma}_{u}$, as well as those in $\boldsymbol{\mu}_{X}$ and $\boldsymbol{\Sigma}_{X}$, are identified. The constraint that

$$
\sum_{j=1}^{J} X_{j}=0
$$

hints that only the reduced mean and covariance structure of the parameters are identified. That is, only parameters in $\mathbf{C} \boldsymbol{\mu}_{u}$ and $\mathbf{C} \boldsymbol{\Sigma}_{u} \mathbf{C}^{\prime}$ can be possibly identified, where $\mathbf{C}$ is a contrast matrix. In particular, here we choose the contrast matrix $\mathbf{C}$, of which the $j$ th row represents the contrast between the $j$ th and the average utility of all alternatives. That is, $\mathbf{C}$ is the submatrix of the matrix $\mathbf{I}-(1 / J) \mathbf{1} \mathbf{1}^{\prime}$ with the exclusion of its last row. For example, for $J=3$,

$$
\mathbf{C}=\left[\begin{array}{ccc}
\frac{2}{3} & -\frac{1}{3} & -\frac{1}{3} \\
-\frac{1}{3} & \frac{2}{3} & -\frac{1}{3}
\end{array}\right] .
$$

To conclude, instead of $\boldsymbol{\mu}_{u}$ and $\boldsymbol{\Sigma}_{u}$ or $\boldsymbol{\mu}_{X}$ and $\boldsymbol{\Sigma}_{X}$, the reduced parameters are $\boldsymbol{\mu}_{X(-J)}$ and $\boldsymbol{\Sigma}_{X(-J)}$ for the unconstrained models. Because of similarity in the comparative nature between the proposed paradigm for approval voting and those for paired comparisons and ranking, the identification constraints necessary for estimating their model parameters are similar (Tsai, 2000, 2003; P. L. H. $\mathrm{Yu}, 2000)$. Böckenholt and Tsai (2001) further showed how one among all possible variance-covariance matrices of the utilities $\boldsymbol{\Sigma}_{u}$ can be obtained from the estimates of the reduced variance-covariance matrix $\boldsymbol{\Sigma}_{X(-J)}$.

Thurstone (1927) introduced the notions of Case V and Case III models in which the evaluations or random utilities of the candidates by the judges are assumed to be uncorrelated with equal and unequal variabilities, respectively. In this case, it is more straightforward to impose such constraints on the covariance matrix $\boldsymbol{\Sigma}_{u}$ of $\mathbf{U}$, rather than on the reduced covariance matrix $\boldsymbol{\Sigma}_{X(-J)}$.

\section{ESTIMATION AND MODEL FIT}

To find the estimates of $\boldsymbol{\mu}_{X(-J)}$ and $\boldsymbol{\Sigma}_{X(-J)}$ that maximize the observed likelihood $\Pi_{i} \operatorname{Pr}\left(\mathbf{W}_{i}\right)$, one needs to evaluate $\operatorname{Pr}\left(\mathbf{W}_{i}\right)$ such that

$$
\begin{aligned}
& \operatorname{Pr}\left(\mathbf{W}_{i}\right)=\int_{a_{1}}^{b_{1}} \int_{a_{2}}^{b_{2}} \cdots \int_{a_{J}}^{b_{J}} f\left(\mathbf{y}_{i}\right) d \mathbf{y}_{i}, \\
& \text { where } \begin{cases}a_{j}=\delta, b_{j}=\infty, & \text { if } W_{i j}=1, \\
a_{j}=-\infty, b_{j}=\delta, & \text { if } W_{i j}=0,\end{cases}
\end{aligned}
$$

where $f$ is the $J$-dimensional multivariate-normal density function of $\mathbf{Y}_{i}$. That is, to obtain the probability for each response pattern $\mathbf{W}_{i}$, a high-dimensional integration is necessary, and the bounds of integration for each latent judgment $Y_{i j}$ is determined according to Equation 11. For example, if $W_{i j}=1$, we have $Y_{i j} \geq \delta$, and the two bounds $a_{j}$ and $b_{j}$ of integration for $Y_{i j}$ are, therefore, from $a_{j}=\delta$ to $b_{j}=\infty$.

Although the numerical maximization of the likelihood function by full maximum-likelihood methods as above is straightforward, it requires the computation of the joint probabilities for the approval voting outcomes. We can easily see that as the number of alternatives increases, the evaluation of the likelihood above through multiple integrations would become cumbersome. For example, the multidimensional integrals are still tractable and can be evaluated numerically for $J \leq 6$, but for larger $J$, numerical integration becomes computationally intractable, and other methods such as simulation-based or limitedinformation (LI) algorithms are better suited to effectively estimate the model parameters. The use of an LI estimator is perhaps favorable because it can be directly adopted and is computationally faster than simulation-based methods, especially when $J$ is large (Maydeu-Olivares \& Böckenholt, 2005).

The LI method plays a critical role in modeling categorical response data in the SEM literature (Christoffersson, 1975; Jöreskog \& Sörbom, 1996; Lee, Poon, \& Bentler, 1995; Maydeu-Olivares, 1999, 2001; B. Muthén, 1978, 1984; Olsson, 1979) and can be performed readily with the popular SEM statistical package Mplus (B. O. Muthén \& Muthén, 2007). In this study, a two-stage LI estimation method with a diagonally weighted least squares (WLS) function for minimization at the second stage is used for estimating model parameters (B. Muthén, 1984; B. Muthén, du Toit, \& Spisic, 1997).

To assess the goodness of fit of the models, Satorra and Bentler (1994) and B. Muthén (1993) showed that, with proper scaling, the Satorra-Bentler adjusted $T$ statistic can be used both for the assessment of overall fit of the models and for fit comparisons of nested models (Satorra \& Bentler, 1994). In particular, the scaled $\chi^{2}$-difference test statistic should be employed for comparing two nested models (Satorra, 2000; Satorra \& Bentler, 2001). For the mean- and variance-adjusted WLS estimator (WLSMV), the DIFFTEST option has been implemented in Mplus to facilitate the testing of two nested models (B. O. Muthén et al., 1997, B. Muthén \& Muthén, 2007). In addition, RMSEA also has been shown to be effective in assessing model fit for models using WLSMV for data with categorical and continuous outcomes (C.-Y. Yu, 2002; C.-Y. Yu $\&$ Muthén, 2001) and thus will be used in the subsequent analysis in this article.

\section{SIMULATION STUDIES}

In this section, we present the results of several simulation studies to evaluate the performance of WLSMV in 
estimating the model parameters. We first compare the maximum likelihood estimates (MLE) obtained by numerical integration using quadrature approximation with the WLSMV estimates for $J=3$ and $J=4$ with $n=300$, 1,000 , and 5,000 judges. The parameters used to generate the approval voting data are as follows:

With $J=3$,

$$
\begin{aligned}
& \boldsymbol{\mu}_{u}=\left(\begin{array}{c}
-1.5 \\
0 \\
2
\end{array}\right), \Sigma_{u}=\left(\begin{array}{lll}
3 & 0 & 3 \\
0 & 6 & 3 \\
3 & 3 & 9
\end{array}\right), \\
& \text { and } \delta=0.5,
\end{aligned}
$$

and accordingly, we have

$$
\begin{gathered}
\boldsymbol{\mu}_{X(-3)}=\left(\begin{array}{c}
-1.5 \\
0
\end{array}\right), \Sigma_{X(-3)}=\left(\begin{array}{cc}
\frac{7}{3} & -\frac{5}{3} \\
-\frac{5}{3} & \frac{10}{3}
\end{array}\right), \\
\text { and } \delta=0.5 .
\end{gathered}
$$

With $J=4$,

$$
\boldsymbol{\mu}_{u}=\left(\begin{array}{c}
-1.5 \\
0 \\
1.5 \\
2
\end{array}\right), \Sigma_{u}=\left(\begin{array}{lllc}
3 & 0 & 3 & 6 \\
0 & 6 & 3 & 3 \\
3 & 3 & 9 & 6 \\
6 & 3 & 6 & 12
\end{array}\right),
$$

and $\delta=0.5$,

and accordingly, we have

$$
\begin{aligned}
\boldsymbol{\mu}_{X(-4)} & =\left(\begin{array}{c}
-2 \\
-0.5 \\
1
\end{array}\right), \Sigma_{X(-4)}=\left(\begin{array}{ccc}
1.5 & -1.5 & -0.75 \\
-1.5 & 4.5 & -0.75 \\
-0.75 & -0.75 & 3
\end{array}\right), \\
& \text { and } \delta=0.5 \text {. }
\end{aligned}
$$

For each set of population parameters, 100 approval voting data sets were generated. Tables 1 and 2 contain the identified parameters, as well as the means and standard deviations of both the WLSMV and MLE estimates for $J=3$ and $J=4$, respectively. As was to be expected, the mean estimates exhibit much less bias than do the estimates of the variance components. The standard deviations of the variances and covariances are large for the case of $n=300$, indicating that, with only 300 approval votes, it is diffi- cult to obtain precise estimates of the variance-covariance structure of the latent utilities for both estimators. Furthermore, for the case of $J=4$, even with a sample size as large as 1,000 , the estimates for the variance in particular still exhibit measurable bias, and their respective standard deviations are also notable. However, as the sample size increases to as large as 5,000, the bias and standard deviations of the estimates are greatly reduced.

We note that the positive bias of the variance-covariance estimates become greater when $J$ increases from 3 to 4 . Equally important, the respective standard deviations of these parameter estimates also increase substantially in comparison with the case of $J=3$, indicating that the more voting alternatives we have in the choice set, the larger the number of all the possible patterns there is, and therefore, a large sample size would be necessary to obtain precise estimates of the variance-covariance parameters of the latent utilities. For example, on the basis of these simulation results, we would suggest that a sample size of at least 500 votes is necessary and recommended to yield reliable and efficient estimates for $J=4$. As the number of candidates in the approval voting choice set increases, the sample size required for obtaining reliable and efficient estimates would increase accordingly. Fortunately, the approval voting data sets reported in the literature usually contain samples of large sizes ranging from a few hundred to around fifty thousand where an approval voting task was used for elections. In addition, the bias and standard deviations of the WLSMV and the MLE estimates appear to be very comparable, although the computation time required for getting WLSMV estimates using the LI method is shorter, in the magnitude of minutes for the case with $J=4$, than that for obtaining MLE estimates using the quadrature method on an Intel Centrino Duo $1 \mathrm{G}$ processor. Overall, these simulation results indicate that WLSMV estimation serves as an alternative to the estimation of approval voting models under the Thurstonian framework.

\section{APPLICATIONS}

\begin{tabular}{|c|c|c|c|c|c|c|c|c|c|c|c|c|c|}
\hline \multirow[b]{4}{*}{ Parameters } & \multirow[b]{4}{*}{ True } & \multicolumn{12}{|c|}{$n$} \\
\hline & & \multicolumn{4}{|c|}{300} & \multicolumn{4}{|c|}{1,000} & \multicolumn{4}{|c|}{5,000} \\
\hline & & \multicolumn{2}{|c|}{ WLSMV } & \multicolumn{2}{|c|}{ MLE } & \multicolumn{2}{|c|}{ WLSMV } & \multicolumn{2}{|c|}{ MLE } & \multicolumn{2}{|c|}{ WLSMV } & \multicolumn{2}{|c|}{ MLE } \\
\hline & & $M$ & $S D$ & $M$ & $S D$ & $M$ & $S D$ & $M$ & $S D$ & $M$ & $S D$ & $M$ & $S D$ \\
\hline$\mu_{X_{1}}$ & -1.50 & -1.56 & 0.41 & -1.57 & 0.43 & -1.50 & 0.17 & -1.50 & 0.16 & -1.50 & 0.07 & -1.50 & 0.07 \\
\hline$\mu_{X_{2}}$ & 0.00 & 0.01 & 0.18 & 0.01 & 0.18 & 0.00 & 0.08 & 0.00 & 0.08 & 0.00 & 0.04 & 0.00 & 0.04 \\
\hline$\sigma_{X_{1}}^{2}$ & 2.33 & 3.04 & 2.49 & 3.07 & 2.61 & 2.38 & 0.68 & 2.35 & 0.61 & 2.33 & 0.29 & 2.34 & 0.29 \\
\hline$\sigma_{X_{2}}^{2}$ & 3.33 & 4.02 & 2.67 & 4.05 & 2.79 & 3.37 & 0.80 & 3.33 & 0.75 & 3.34 & 0.36 & 3.35 & 0.37 \\
\hline$\sigma_{X_{1} X_{2}}$ & -1.67 & -2.19 & 1.84 & -2.21 & 1.95 & -1.67 & 0.50 & -1.64 & 0.45 & -1.67 & 0.22 & -1.67 & 0.22 \\
\hline$\delta$ & 0.50 & 0.54 & 0.18 & 0.54 & 0.18 & 0.50 & 0.06 & 0.50 & 0.06 & 0.50 & 0.03 & 0.50 & 0.03 \\
\hline
\end{tabular}

\section{TIMS E1 Revisited}

We first analyze the TIMS E1 data set for estimates comparison. The data set TIMS E1 was originally reported

Table 1

Identified Parameter Values and the Means and Standard Deviations of Their Respective WLSMV and MLE Estimates for $J=3$ With $n=300,1,000$, and 5,000 (With $\sigma_{\epsilon}^{2}=1$ )

Note-The $S D$ s are the standard deviations of the estimates obtained in the 100 replications. 
Table 2

Identified Parameter Values and the Means and Standard Deviations of Their Respective WLSMV and MLE Estimates for $J=4$ with $n=300,1,000$, and 5,000 (With $\sigma_{\epsilon}^{2}=1$ )

\begin{tabular}{|c|c|c|c|c|c|c|c|c|c|c|c|c|c|}
\hline \multirow[b]{4}{*}{ Parameters } & \multirow[b]{4}{*}{ True } & \multicolumn{12}{|c|}{$n$} \\
\hline & & \multicolumn{4}{|c|}{300} & \multicolumn{4}{|c|}{1,000} & \multicolumn{4}{|c|}{5,000} \\
\hline & & \multicolumn{2}{|c|}{ WLSMV } & \multicolumn{2}{|c|}{ MLE } & \multicolumn{2}{|c|}{ WLSMV } & \multicolumn{2}{|c|}{ MLE } & \multicolumn{2}{|c|}{ WLSMV } & \multicolumn{2}{|c|}{ MLE } \\
\hline & & $M$ & $S D$ & $M$ & $S D$ & $M$ & $S D$ & $M$ & $S D$ & $M$ & $S D$ & $M$ & $S D$ \\
\hline$\mu_{X_{1}}$ & -2.00 & -2.25 & 1.54 & -2.63 & 1.55 & -2.10 & 0.40 & -2.11 & 0.43 & -2.01 & 0.14 & -2.02 & 0.13 \\
\hline$\mu_{X_{2}}$ & -0.50 & -0.56 & 0.25 & -0.56 & 0.33 & -0.53 & 0.14 & -0.53 & 0.15 & -0.51 & 0.06 & -0.51 & 0.06 \\
\hline$\mu_{X_{3}}$ & 1.00 & 1.13 & 0.88 & 1.29 & 0.72 & 1.06 & 0.23 & 1.07 & 0.25 & 1.01 & 0.08 & 1.01 & 0.08 \\
\hline$\sigma_{X_{1}}^{2}$ & 1.50 & 3.68 & 17.60 & 4.92 & 10.02 & 1.83 & 1.20 & 1.89 & 1.34 & 1.55 & 0.38 & 1.55 & 0.36 \\
\hline$\sigma_{X_{2}}^{2}$ & 4.50 & 7.98 & 25.24 & 9.76 & 13.69 & 5.11 & 2.10 & 5.22 & 2.31 & 4.60 & 0.64 & 4.61 & 0.61 \\
\hline$\sigma_{X_{3}}^{2}$ & 3.00 & 5.48 & 19.41 & 4.50 & 4.90 & 3.43 & 1.56 & 3.51 & 1.72 & 3.06 & 0.41 & 3.07 & 0.39 \\
\hline$\sigma_{X_{1} X_{2}}$ & -1.50 & -3.03 & 11.97 & -4.91 & 9.64 & -1.75 & 0.95 & -1.81 & 1.07 & -1.55 & 0.32 & -1.56 & 0.31 \\
\hline$\sigma_{X_{1} X_{3}}$ & -0.75 & -1.70 & 8.39 & -1.30 & 3.02 & -0.88 & 0.58 & -0.91 & 0.63 & -0.77 & 0.17 & -0.77 & 0.16 \\
\hline$\sigma_{X_{2} X_{3}}$ & -0.75 & -1.48 & 6.11 & -1.15 & 0.93 & -0.85 & 0.47 & -0.86 & 0.50 & -0.77 & 0.15 & -0.77 & 0.15 \\
\hline$\delta$ & 0.50 & 0.59 & 0.52 & 0.75 & 0.57 & 0.53 & 0.12 & 0.54 & 0.14 & 0.51 & 0.05 & 0.51 & 0.05 \\
\hline
\end{tabular}

Note-The $S D$ s are the standard deviations of the estimates obtained in the 100 replications.

in Fishburn and Little (1988) and was tested in Regenwetter and Grofman (1998) and Regenwetter et al. (2007). Each election had three candidates running for a single seat. To examine the validity of our estimation method, we first compare the MLE and WLSMV estimates of the identified parameters for the model with $\delta=0$. Both sets of parameter estimates yield similar estimates of the identified parameters. For illustrative purposes, in Table 3, we report both the MLE and the WLSMV estimates and their respective standard errors .

Previous analysis of the TIMS E1 data by Regenwetter et al. (2007) showed that a single-population IP-RUM model provided a reasonable fit to the subset of data, excluding the none and all response patterns. Therefore, the IP-RUM heuristic serves as a plausible underlying mechanism for the approval voting process in these data. Here, we analyze the complete TIMS E1 data, using Mplus to check whether a model with an occasion-specific term $\epsilon$ - that is, Equation 9-is suitable. Table 4 shows a summary of the results, indicating that the models with the inclusion of $\epsilon$ again provide a good fit to the complete data $[T(1)=$ $0.810, p=.3681$, and RMSEA $<.001]$.

To check whether the assumption of uncorrelated utilities of the candidates holds among the judges, we also considered models with a Case $\mathrm{V}$ representation for the covariance matrix $\boldsymbol{\Sigma}_{u}\left(M_{4}\right.$ and $\left.M_{5}\right)$ in Table 4 . The results imply that a Case V structure for $\boldsymbol{\Sigma}_{u}$ is too strong an assumption with or without the inclusion of the threshold $\delta$, with the $p$ values for $M_{4}$ and $M_{5}$ both less than .001 . Moreover, we considered model $M_{2}$, which is not a Case $\mathrm{V}$, but with

$$
\begin{aligned}
& \operatorname{Var}\left(U_{1}-\frac{U_{1}+U_{2}+U_{3}}{3}\right) \\
& \quad=\operatorname{Var}\left(U_{2}-\frac{U_{1}+U_{2}+U_{3}}{3}\right)\left(\text { i.e., } \sigma_{X_{1}}^{2}=\sigma_{X_{2}}^{2}\right) .
\end{aligned}
$$

The constraint of $M_{3}$ frees up onedegree of freedom for the model and therefore makes it possible to access the necessity of the threshold parameter $\delta$. On the basis of the chi-square test for difference testing (DIFFTEST) of the two nested models $M_{1}$ and $M_{2}$, we conclude that $M_{2}$ is preferred to $M_{1}[T(1)=1.407, p=.2356]$. Moreover, we consider model $M_{3}$ where a nonzero threshold parameter $\delta$ is assumed in the underlying mechanism and $M_{3}$ also provides a reasonable fit to the data. However, with a threshold estimate $[\hat{\delta}=-0.009(0.024)]$ not significantly different from zero and with a nonsignificant DIFFTEST result $[T(1)=0.139, p=.7088]$, model $M_{3}$ is thought to provide no better fit than model $M_{2}$. To sum up, with the inclusion of $\epsilon$, model $M_{2}$ provides the most parsimonious fit to the complete TIMS E1 data and yields a reasonable underlying mechanism for the voting process. The parameter estimates and their respective standard errors (in the parentheses) for $M_{2}$ are

$$
\hat{\boldsymbol{\mu}}_{X(-3)}=(-0.946(0.058) \quad 0.621(0.051))^{\prime}
$$

and

$$
\hat{\Sigma}_{X(-3)}=\left(\begin{array}{cc}
1.252(0.190) & -0.356(0.116) \\
-0.356(0.116) & 1.252(0.190)
\end{array}\right) .
$$

Note that model $M_{2}$ can now account for both the all and none responses. From the estimates of $M_{2}$, we see that, if we would like to account for all the responses in the underlying decision heuristic, the within-judge variability $\left[\operatorname{Var}\left(\epsilon_{j}\right)=1\right]$ is substantial in comparison with the between-judge variability $\hat{\sigma}_{X_{1}}^{2}=\hat{\sigma}_{X_{2}}^{2}=1.252(0.190)$.

Table 3

MLE and WLSMV Estimates and Their Associated Standard Errors Obtained for the Complete TIMS E1 Data Set With $\boldsymbol{n}=\mathbf{1 , 5 6 7}$

\begin{tabular}{crrrrr}
\hline & \multicolumn{2}{c}{ MLE } & & \multicolumn{2}{c}{ WLSMV } \\
\cline { 2 - 3 } \cline { 5 - 6 } Parameters & \multicolumn{1}{c}{$M$} & $S E$ & & $M$ & $S E$ \\
\hline$\mu_{X_{1}}$ & -0.935 & 0.057 & & -0.934 & 0.058 \\
$\mu_{X_{2}}$ & 0.621 & 0.051 & & 0.622 & 0.051 \\
$\sigma_{X_{1}}^{2}$ & 1.149 & 0.198 & & 1.142 & 0.196 \\
$\sigma_{X_{2}}^{2}$ & 1.325 & 0.211 & & 1.344 & 0.217 \\
$\sigma_{X_{1} X_{2}}$ & -0.360 & 0.116 & -0.358 & 0.116 \\
\hline
\end{tabular}


Table 4

Analysis of TIMS E1 Data

\begin{tabular}{llcccc}
\hline Model & \multicolumn{1}{c}{ Constraints } & $T$ Statistic & $d f$ & $p$ Value & RMSEA \\
\hline$M_{1}$ & $\Sigma_{X(-J)}$ unconstrained & 0.810 & 1 & .3681 & .000 \\
$M_{2}$ & $\sigma_{X_{1}}=\sigma_{X_{2}}, \delta=0$ & 2.373 & 2 & .3053 & .011 \\
$M_{3}$ & $\sigma_{X_{1}}=\sigma_{X_{2}}, \delta \neq 0$ & 1.781 & 1 & .1820 & .022 \\
$M_{4}$ & Case V, $\delta=0$ & 17.195 & 3 & .0006 & .055 \\
$M_{5}$ & Case V, $\delta \neq 0$ & 14.536 & 2 & .0007 & .063 \\
\hline
\end{tabular}

From the relation of $X_{3}=-X_{1}-X_{2}$, we can obtain the parameter estimates for all the candidates

$$
\hat{\boldsymbol{\mu}}_{X}=\left(\begin{array}{lll}
-0.946 & 0.621 & 0.325
\end{array}\right)^{\prime}
$$

and

$$
\hat{\Sigma}_{X}=\left(\begin{array}{ccc}
1.252 & -0.356 & -0.896 \\
-0.356 & 1.252 & -0.896 \\
-0.896 & -0.896 & 1.792
\end{array}\right) .
$$

The ordering of the values of the $\hat{\mu}$ s indicates that Candidate 2 is, on average, the most preferred, whereas Candidate 1 is the least preferred in the complete TIMS E1 data, and the overall conclusion on preference ordering is consistent with that in Regenwetter et al. (2007). Moreover, the ordering of the candidates' mean utilities that $\mu_{U_{2}}>$ $\mu_{U_{3}}>\mu_{U_{1}}$ is also consistent with those of the Borda order (231) and of possible social welfare order (231 or 321) (Regenwetter \& Grofman, 1998).

With a larger variance of $\hat{\sigma}_{X_{3}}^{2}=1.792$, more variability is implied for the deviation utility for Candidate 3 ,

$$
X_{3}=U_{3}-\frac{U_{1}+U_{2}+U_{3}}{3},
$$

than for the other two candidates in the judgment process. Moreover, the correlation matrix of $X \mathrm{~s}$,

$$
\hat{\boldsymbol{\rho}}_{X}=\left(\begin{array}{ccc}
1 & -0.284 & -0.598 \\
-0.284 & 1 & -0.598 \\
-0.598 & -0.598 & 1
\end{array}\right),
$$

shows that the deviation utilities for Candidates 1 and 2 is less correlated in absolute magnitude with $\hat{\rho}_{12}=-0.284$ than are the other correlations. For illustration, we include in Appendix A the input syntax file of $M_{2}$ for the TIMS E1 data.

Regenwetter et al. (2007) considered a variety of different heuristics other than IP-RUM in understanding the underlying mechanism of approval voting, although their model testing was limited to data sets with three candidates only. In particular, even with the IEEE election data, which originally contained votes for four candidates from the presidential election of the Institute of Electrical and Electronics Engineers (Brams \& Nagel, 1991), Regenwetter et al. chose and analyzed a subset of approval voting responses of only three out of the four candidates. To illustrate the usefulness of formulating the models under the SEM framework, we analyzed the complete IEEE data as an empirical example with four alternatives in the approval choice set. The great advantage of the proposed models over the previous IP-RUM model proposed by Regenwetter et al. is that the estimation by limited information methods permits the analysis of approval voting data with an arbitrary number of candidates.

\section{IEEE Revisited}

We analyze the complete IEEE data, and a summary of the results is reported in Table 5. The comparison between $M_{1}$ and $M_{2}$ indicates that the latter model with the inclusion of a nonzero threshold $\delta$ provides a much better fit to the data. More specifically, $M_{2}$ shows a reasonable fit to the data based on the obtained statistics $[T(1)=1.988$, $p=.1586$, RMSEA $=.004]$. Each of the models $M_{2}$ to $M_{4}$ restricts one of the covariances between the $X \mathrm{~s}$ to be zero. Among the three models, only $M_{2}$ yields acceptable fit statistics. To see whether further simplification is possible for $M_{2}$, the DIFFTEST result between $M_{2}$ and $M_{5}$ shows that the former model fits much better. With the inclusion of $\delta$, model $M_{2}$ yields a reasonable underlying mechanism for the voting process.

The parameter estimates and their respective standard errors (in the parentheses) for $M_{2}$ are

$$
\begin{aligned}
& \hat{\boldsymbol{\mu}}_{X(-4)} \\
& =\left(\begin{array}{lll}
1.282(0.051) & 0.476(0.025) & -2.521(0.104)
\end{array}\right)^{\prime}, \\
& \hat{\Sigma}_{X(-4)} \\
& =\left(\begin{array}{ccc}
8.021(0.690) & 0 & -8.877(0.747) \\
0 & 3.375(0.303) & -3.049(0.275) \\
-8.877(0.747) & -3.049(0.275) & 19.362(1.635)
\end{array}\right) \text {, }
\end{aligned}
$$

and

$$
\hat{\delta}=1.261(0.051) .
$$

In other words, from $\hat{\boldsymbol{\mu}}_{X(-4)}$, we would conclude that $\mu_{U_{1}}>$ $\mu_{U_{4}}>\mu_{U_{2}}>\mu_{U_{3}}$, indicating that Candidate 1 is, on average, the most favorite candidate, followed by Candidate 4 , Candidate 2, and Candidate 3 at last. If we examine only the ordering of $C_{1}, C_{2}$, and $C_{3}$, because those are the ones analyzed in the previous study, the attained ordering of the

Table 5

Analysis of IEEE Data

\begin{tabular}{clrrrc}
\hline Model & \multicolumn{1}{c}{ Constraints } & \multicolumn{1}{c}{$T$ Statistic } & $d f$ & $p$ Value & RMSEA \\
\hline$M_{1}$ & $\delta=0$ & $31,802.012$ & 1 & $<.0001$ & 0.755 \\
$M_{2}$ & $\delta \neq 0 ; \sigma_{X_{1} X_{2}}=0$ & 1.988 & 1 & .1586 & 0.004 \\
$M_{3}$ & $\delta \neq 0 ; \sigma_{X_{1} X_{3}}=0$ & $15,059.775$ & 1 & $<.0001$ & 0.519 \\
$M_{4}$ & $\delta \neq 0 ; \sigma_{X_{2} X_{3}}=0$ & $1,877.479$ & 1 & $<.0001$ & 0.183 \\
$M_{5}$ & $\delta \neq 0 ; \sigma_{X_{1} X_{2}}=0, \mu_{X_{2}}=0$ & $1,100.045$ & 2 & $<.0001$ & 0.099 \\
\hline
\end{tabular}


candidates' mean utilities that $\mu_{U_{1}}>\mu_{U_{2}}>\mu_{U_{3}}$ is consistent with those of the Borda order (123) and, possibly, of the social welfare order $(123,132$, or cycle) (Regenwetter \& Grofman, 1998).

Furthermore, the level of variabilities for $X_{1}, X_{2}$, and $X_{3}$, with $\hat{\sigma}_{X_{1}}^{2}=8.021(0.690), \hat{\sigma}_{X_{2}}^{2}=3.375(0.303)$, and $\hat{\sigma}_{X_{3}}^{2}=$ 19.362 (1.635), are quite different where $X_{3}$ exhibits much higher level of variability. In addition, the fact that

$$
\begin{aligned}
\operatorname{Cov}\left(X_{1}, X_{2}\right) & =\operatorname{Cov}\left(U_{1}-\frac{\sum_{j=1}^{4} U_{i j}}{4}, U_{2}-\frac{\sum_{j=1}^{4} U_{i j}}{4}\right) \\
& =0
\end{aligned}
$$

indicates independence between the latent evaluations of $U_{1}$ and $U_{2}$ while using the average utilities as the reference. Furthermore, we have $\hat{\delta}=1.261(0.051)$, significantly different from 0 . In other words, on average, a candidate needs to have an utility greater than the average utility by 1.261 to be approved. The Mplus input file of $M_{2}$ for IEEE data is reported in Appendix B.

Analyses of the two empirical data sets, TIMS E1 and IEEE, show that in both cases the ordering of the candidates' mean utilities is consistent with those of the Borda order and some possible social welfare order. That is, the estimates of our proposed model seem to yield the same winner as those from other social choice criteria. However, theoretical and simulation studies would still be necessary to investigate whether this is in general the case and under what situations the proposed models will or will not arrive at the same conclusion as those of others.

\section{CONCLUSION}

In this article, we embed the random utility models of approval voting data under the SEM framework in order to investigate the underlying mechanism of the choice process and, therefore, provide a means to understand the voting behavior. By doing so, we are now able to extend previous approaches in such a way as to analyze data with any number of alternatives in the choice set. In particular, the complete data, including the none and all approval patterns, can be analyzed and, therefore, provide an account for all the response patterns in the choice behavior. Moreover, the approval voting mechanism can also be used for a consideration set formulation in marketing or consumer behavior research, where both the none and all responses are always included in the analysis.

As a first attempt to account for the none and all responses in the complete approval voting data, our model introduces the occasion-specific error term, and its inclusion implies that the two patterns happen merely due to random chances. In addition, the strong assumption of homogeneity for the occasion-specific error terms across all the choice alternatives induces the property of equal probabilities for these two responses, although this might not always be the case in empirical data. Thus, more investigation is needed to obtain a systematic account for the none and all responses in approval voting behavior. Collecting and studying data on situations or experimen- tal settings where a single judge is asked to give approval voting of the same choice set multiple times is perhaps the next step to better understanding and characterizing the choice process.

For data with more than four choice items, we propose the use of a limited-information estimation method to facilitate the estimation of such models that would otherwise require high-dimensional integration. However, such an approach does have a shortcoming in that the present approach can be adopted to analyze data only from a single population. On the basis of past research and our analysis of a number of other empirical data sets, singlepopulation models often do not conform to the true underlying mechanism in the data, whereas a mixture model is necessary to capture individual differences among the voters. For example, Regenwetter et al. (2007) found that it is necessary to consider several different types of heuristics in order to accommodate interindividual variability and to make better sense of the empirical variability in ballot-casting behavior. Thus, more efficient estimation methods for mixture models of data with a moderate to large number of alternatives or candidates are very much in need. However, this outlook of future work should not distract from the fact that the analysis of approval voting data with more than five or six candidates is now accessible to researchers, and hopefully, this work will encourage more research on approval voting and similar subset choice paradigms.

\section{AUTHOR NOTE}

This research was supported by the National Science Council of Taiwan (NSC 94-2413-H-003-024 and NSC 95-2413-H-003-004). The author is very grateful to the reviewers for their constructive comments, which led to substantive improvement in the manuscript. Correspondence concerning this article should be addressed to R.-C. Tsai, Department of Mathematics, National Taiwan Normal University, No. 88, Sec. 4, TingChou Rd., Taipei 116, Taiwan (e-mail: rtsai@math.ntnu.edu.tw).

\section{REFERENCES}

Bian, X., \& Moutinho, L. (2009). An investigation of determinants of counterfeit purchase consideration. Journal of Business Research, 62, 368-378.

BöcKenholt, U., \& TsaI, R. (2001). Individual differences in paired comparison data. British Journal of Mathematical \& Statistical Psychology, 54, 265-277.

Brams, S. J., \& Fishburn, P. C. (1983). Approval voting. Boston: Birkhäuser.

Brams, S. J., \& Nagel, J. H. (1991). Approval voting in practice. Public Choice, 71, 1-17.

Christoffersson, A. (1975). Factor analysis of dichotomized variables. Psychometrika, 40, 5-32.

Eliaz, K., \& SPIEGLER, R. (2009). Consideration sets and competitive marketing (CEPR Discussion Paper 7456). London: Centre for Economic Policy Research.

Falmagne, J. C., \& Regenwetter, M. (1996). A random utility model for approval voting. Journal of Mathematical Psychology, 40, 152159

Fishburn, P. C., \& Little, J. D. C. (1988). An experiment in approval voting. Management Science, 34, 555-568.

Haubl, G., \& Trifts, V. (2000). Consumer decision making in online environments. Marketing Science, 19, 4-21.

JöRESKOG, K. G., \& SöRBOM, D. (1996). LISREL 8: User's reference guide. Chicago: Scientific Software International.

Lee, S. Y., Poon, W. Y., \& Bentler, P. M. (1995). A two-stage estimation of structural equation models with continuous and polytomous 
variables. British Journal of Mathematical \& Statistical Psychology, 48, 339-358.

Marley, A. A. J. (1993). Aggregation theorems and the combination of probabilistic rank orders. In D. E. Critchlow, M. A. Fligner, \& J. S. Verducci (Eds.), Probability models and data analysis for ranking data: Lecture notes in statistics (pp. 216-240). New York: Springer.

Marley, A. A. J. (2002). Random utility models and their applications: Recent developments. Mathematical Social Sciences, 43, 289-302.

MAYdeu-Olivares, A. (1999). Thurstonian modeling of ranking data via mean and covariance structure analysis. Psychometrika, 64, 325-340.

Maydeu-Olivares, A. (2001). Limited information estimation and testing of Thurstonian models for paired comparison data under multiple judgment sampling. Psychometrika, 66, 209-228.

Maydeu-Olivares, A., \& Böckenholt, U. (2005). Structural equation modeling of paired-comparison and ranking data. Psychological Methods, 10, 285-304.

MerRILl, S. (1979). Approval voting: "Best buy" method for multicandidate elections? Mathematics Magazine, 52, 98-102.

MuthéN, B. [O.] (1978). Contributions to factor analysis of dichotomous variables. Psychometrika, 43, 551-560.

MuthéN, B. [O.] (1984). A general structural equation model with dichotomous, ordered categorical, and continuous latent variable indicators. Psychometrika, 49, 115-132.

Muthén, B. [O.] (1993). Goodness of fit with categorical and other non-normal variables. In K. A. Bollen \& J. S. Long (Eds.), Testing structural equation models (pp. 205-234). Newbury Park, CA: Sage.

Muthén, B. [O.], DU ToIT, S. H. C., \& SPISIC, D. (1997). Robust inference using weighted least squares and quadratic estimating equations in latent variable modeling with categorical and continuous outcomes. Unpublished manuscript.

Muthén, B. O., \& MuthéN, L. K. (2007). Mplus: Statistical analysis with latent variables (Version 4.2). Los Angeles: Statmodel.

Muthén, L. K., \& MuthéN, B. O. (2007). Mplus user's guide (5th ed.). Los Angeles: Muthén \& Muthén. (Original work published 1998)

Olsson, U. (1979). Maximum likelihood estimation of the polychoric correlation coefficient. Psychometrika, 44, 443-460.

Paap, R., van Nierop, E., van Heerde, H. J., Wedel, M., Franses, P. H., \& Alsem, J. A. (2005). Consideration sets, intentions and the inclusion of "don't know" in a two-stage model for voter choice. International Journal of Forecasting, 21, 53-71.

PunJ, G., \& MoORe, R. (2009). Information search and consideration set formation in a Web-based store environment. Journal of Business Research, 62, 644-650.

Regenwetter, M., \& Grofman, B. (1998). Approval voting, Borda winners, and Condorcet winners: Evidence from seven elections. Management Science, 44, 520-533.

Regenwetter, M., Ho, M.-H. R., \& Tsetlin, I. (2007). Sophisticated approval voting, ignorance priors, and plurality heuristics: A behav- ioral social choice analysis in a Thurstonian framework. Psychological Review, 114, 994-1114.

SATORRA, A. (2000). Scaled and adjusted restricted tests in multi-sample analysis of moment structures. In D. D. H. Heijmans, D. S. G. Pollock, \& A. Satorra (Eds.), Innovations in multivariate statistical analysis: A festschrift for Heinz Neudecker (pp. 233-247). Dordrecht: Kluwer.

SAtorra, A., \& Bentler, P. M. (1994). Corrections to test statistics and standard errors in covariance structure analysis. In A. von Eye \& C. C. Clogg (Eds.), Latent variable analysis: Applications to developmental research (pp. 399-419). Thousand Oaks, CA: Sage.

Satorra, A., \& Bentler, P. M. (2001). A scaled difference chi-square test statistic for moment structure analysis. Psychometrika, 66, 507514

Shapiro, S., Macinnis, D. J., \& Heckler, S. E. (1997). The effects of incidental ad exposure on the formation of consideration sets. Journal of Consumer Research, 24, 94-104.

Shocker, A. D., Ben-Akiva, M., Boccara, B., \& Nedungadi, P. (1991). Consideration set influences on consumer decision-making and choice: Issues, models, and suggestions. Marketing Letters, $\mathbf{2}$ 181-197.

TAKANE, Y. (1998). Choice model analysis of the "pick any/n" type of binary data. Japanese Psychological Research, 40, 31-39.

Thurstone, L. L. (1927). A law of comparative judgment. Psychological Review, 34, 273-286.

Tillie, J. (1995). Party utility and voting behavior. Amsterdam: Het Spinhuis.

TsaI, R. (2000). Remarks on the identifiability of Thurstonian ranking models: Case V, Case III, or neither? Psychometrika, 65, 233-240.

TsAI, R. (2003). Remarks on the identifiability of Thurstonian paired comparison models under multiple judgment. Psychometrika, 68 , 361-372.

TSAI, R., \& BÖCKENHOLT, U. (2006). Modeling intransitive preferences: A random-effects approach. Journal of Mathematical Psychology, 50, 1-14.

TsaI, R., \& Wu, T.-L. (2004). Analysis of paired comparison data using Mx. Structural Equation Modeling, 11, 73-91.

van der Eijk, C., van der Burg, W., Kroh, M., \& Franklin, M. (2006). Rethinking the dependent variable in voting behavior: On the measurement and analysis of electoral utilities. Electoral Studies, 25, 424-447.

Yu, C.-Y. (2002). Evaluating cutoff criteria of model fit indices for latent variable models with binary and continuous outcomes. Unpublished doctoral dissertation, University of California, Los Angeles.

Yu, C.-Y., \& MuthÉN, B. O. (2001). Evaluation of model fit indices for latent variable models with categorical and continuous outcomes (Tech. Rep.). Los Angeles: University of California.

YU, P. L. H. (2000). Bayesian analysis of order-statistics models for ranking data. Psychometrika, 65, 281-299. 
APPENDIX A

MPLUS Script for Model $M_{2}$ of the TIMS E1 Data Set
